\title{
El $5^{\circ}$ Symposium grafica. La consolidación de un punto de encuentro académico
} y de investigación en diseño gráfico 0

\section{Resumen}

Tic-tac, tic-tac. El tiempo es una máquina imparable de borrar recuerdos. Los días se suceden, los años se acumulan en las estanterías de la memoria y parece que no tengamos tiempos de apreciar lo mejor de lo bueno, lo regular de lo malo y lo malo de lo peor. Todo avanza tan deprisa que no podemos permitirnos saborear el momento. Pero han pasado cinco septiembres, cinco años en los que la revista gráfica y todo el equipo humano que la hace posible organizamos el proyecto del Symposium grafica. El proyecto nació con la esperanza de crear un punto de encuentro para los autores de la revista y los profesores de diseño y publicidad que sienten pasión por la comunicación gráfica y el universo de las imágenes. $Y$ vale la pena pararse un momento parar saborear el éxito del último Symposium.

\section{Palabras Clave}

grafica journal; symposium; diseño gráfico; publicidad; comunicación

\section{ABSTRACT}

The 5th Symposium grafica. The consolidation of an academic and research in graphic design convention

Tic-tac, tic-tac. The time is an unstoppable machine to erase memories. The days follow one another, the years accumulate on the shelves of memory and it seems that we do not have times of perception the best of the good, the regulars of the bad and the bad of the worst. Everything is moving so fast that we cannot afford to svour the moment. Five years have gone, five Septembers, five years of the grafica journal and the entire human team that makes it possible to organize the grafica Symposium project. The project was born with the hope of creating a meeting point for the authors of the journal and the professors of design and advertising who have a passion for graphic communication and the universe of images. And it is worth stopping for a moment to savour the success of the last Symposium.

\section{Keywords}

Grafica journal; symposium; graphic design; advertising; communication
Nunca pudimos imaginar que este congreso académico, nuestro quinto congreso, se convertiría en una cita anual tan bienvenida. Es un placer inmenso reencontrarse cada año con los amigos del año anterior y añadir nuevos compañeros a nuestra comunidad de investigadores en diseño y comunicación. Como director de un grupo de investigación que coorganiza estas jornadas no puedo sentirme más orgulloso. Investigamos para compartir, sin esta comunidad mucho de lo que hacemos carece de sentido.

El formato del Symposium empezó modesto y familiar, como un encuentro entre amigos. Nos sentimos cómodos debatiendo en la facultad de comunicación de BlanquernaUniversitat Ramon Llull (2015) y seguimos con ganas de más en los años siguientes, en la facultad de comunicación de la Universitat Autónoma de Barcelona (2016), en el nuevo edificio de Massana (2017), que prácticamente inauguramos, en la escuela de Artes Gráficas de Salesians de Sarrià (2018) y en la Escola Superior de Relaciones Públicas de la Universitat de Barcelona que nos acogió los días 4, 5 y 6 de septiembre de 2019.

Años tras año, el Symposium grafica se ha convertido en un punto de encuentro de expertos e investigadores, tres días de ponencias, conferencias y mesas redondas en las que cada momento se convierte en un debate, una conversación intensa entre amigos. Que gran ambiente, cinco años después seguimos siendo un grupo de amigos cada vez más numeroso. 
Vale la pena frenar el tic-tac del reloj y recordar que del $5^{\circ}$ Symposium podemos destacar muchas cosas. La primera es la más importante, el equipo formado por Daniel Tena, Ana Entenza y Bea Hontanilla se desvivió por organizar las actividades de manera que el encuentro fluyera sin sobresaltos y la Escola Superior de Relaciones Públicas fue un anfitrión realmente magnífico -gracias Sara Magallón y Antoni Noguero- y el encuentro musical del primer día con un grupo musical, Els Ministrils de La Sabadell, lo que nos permitió brindar por el inicio el Symposium con el alma sensible.

Por otro lado, debemos agradecer a dos grandes maestros, Miquel de Moragas y Joan Costa, por obsequiarnos con dos conferencias que demostraron el valor de estos dos "sabios" de la comunicación. Disfrutamos de inmensa curiosidad que los ha motivado a ser investigadores $y$ al final de sus intervenciones, en cierto modo, también rendimos un homenaje a todo lo que hemos aprendido de ambos. Moragas nos demostró que "es imposible hacer una buena tarea de diseño gráfico sin ser un investigador" y Costa nos explicó que "el esquema hace visible cosas y acontecimientos invisibles". Gracias maestros.

Curiosamente, empezamos la primera jornada con una ponencia muy humanista, todos sabemos que la comunicación gráfica es una manifestación de la cultura urbana y tuvimos la suerte de escuchar al arquitecto Carles Berga hablar del mural que ha diseñado en el nuevo edificio de la Escola Superior de Relaciones Públicas, nos recordó que desde tiempos remotos "los murales han sido cómplices de la representación del ser humano". Curiosamente, Lourdes Pila, de Guayaquil, también abordó la dimensión gráfica de la cultura urbana en su ponencia sobre diseño y patrimonio, Sofía Escudero nos habló de las particularidades culturales en el diseño de packaging de las marcas globales y Erika Alejandra Patiño introdujo el tema del diseñó para el territorio con su ponencia sobre la marca turística de Gualivá en Colombia. Y con esa complicidad humanista nos pusimos a debatir en unas mesas que incidieron en diversos temas que nos preocupan a todos.

Marc Mallafré, Juan Antonio Buenadicha, Josep Rom y Nereida Cea, coincidimos en la presentación e investigaciones relacionadas con el peso de las tecnologías en los procesos profesionales y educativos del diseño gráfico y la publicidad, desde la puesta en escena de montajes multimedia, hasta la importancia de la publicidad programática, pasando por el papel del software de diseño en la formación de los futuros profesionales.

El tema de la enseñanza del diseño, como es habitual en un encuentro de profesores, provocó numerosos debates. Paulina Montenegro y Juan Jesús Arrausi comentaron la importancia de nuevos soportes didácticos, como el workbook, para la formación de los jóvenes diseñadores. Fernando Suárez-Carballo introdujo la preocupación por la evolución de las competencias tecnológicas en los grados en Publicidad en España. Jesús del Hoyo y Francesc Morera coincidieron en la importancia e la digitalización en la formación profesional de diseñadores mediante el aprendizaje basado en talleres. Mientras que Elena Bartomeu nos hacía una demostración práctica del valor de los talleres mediante un proyecto de estudiantes de investigación de la señalización de tránsito. Sheila González habló de tipologías proyectuales en diseño gráfico y Beatríz Martínez-Vilagrasa, Danae Esparza, Toni Llacer, Nicolas Montaretto y Sergi Cotiñas aportaron nueva luz a la importancia de las competencias creativas en diseño, al igual que Lluc Massaguer y Rubén Alcaraz. También en el ámbito de la creatividad, Sara Meléndez y David Roca presentaron su investigador sobre el impacto de los premios creativos en la promoción profesional. La combinación de presentaciones aporta lo mejor del Symposium Grafica, la facilidad de los asistentes a la hora de compartir y cruzar impresiones a partir de sus investigaciones. Es la mejor prueba del valor de estas jornadas como punto de encuentro.

También destinamos un espacio a la historia del diseño y la publicidad. Rosa Fonts recuperó la figura e Marius Serra en el desarrollo de la industria publicitaria de la ciudad de Tarragona, un ejemplo del valor de los pequeños empresarios en la mejora de la cultura del diseño del siglo XX. Enrique Vergara vino desde Chile para presentarnos la evolución de la imagen de la mujer en la publicidad de las revistas femeninas chilenas y Patrícia Lázaro y Alfons González, en una línea paralela, presentó la evolución de la mujer en la publicidad durante la Segunda República española.

Tampoco faltaron los profesionales, un punto de vista que complementa perfectamente el punto de vista académico. Jordi Fernández Cuesta y Jordi Camps hablaron de la "realidad" de su trabajo en publicidad y diseño y, como en años anteriores, el Col-legi Oficial de Disseny Gráfica de Catalunya y el Col-Legi de Publicitaris i Relacions Públiques de Catalunya explicaron los compromisos de estas organizaciones con el impulso de 
la profesión. A nivel académico, Laura Camerino, Cilia Willem y Josep Rom, aportamos un punto de vista complementario a estas exposiciones a partir del análisis de la imagen pública de un profesional la comunicación visual muy conocido, Jordi Labanda.

Como revista académica de investigación compartimos la inquietud por la actividad editorial del sector de las revistas. Patrícia Lázaro, Alfons González, Ana Entenza y Daniel Tena coincidieron en aportar puntos de vista sobre la gestión la calidad y el Data Sharing aplicado a mejorar las investigaciones y las publicaciones. David Roca nos quiso quitar el miedo a la gestión de nuestra estadísticas aplicadas a la investigación, presentando un "manual de estadística aplicada con Jamovi". La pedagogía de Roca intensificó el interés de los investigadores por estas metodologías. Creemos que el conocimiento mejora la pátina del diseño la publicidad, por eso nos gustó escuchar a Marcelo Ghio, editor de Experimenta, en la presentación de los nuevos libros de Joan Costa -"Esquematismo" y "La forma de las ideas"- nos recordó el valor de "leer para diseñar", una frase que sintetiza perfectamente el espíritu de la revista Grafica y el valor de nuestro trabajo.

La gran novedad del $5^{\circ}$ Symposium grafica fue la entrega del Premio Grafica. Un premio que es manifiesto de intenciones del equipo de la revista. Nuestro trabajo tiene sentido porque es un trabajo compartido y el Premio Grafica es un premio que reconoce al autor o autores del artículo más consultado de la revista durante un período concreto (2013-2018). En este caso el galardón fue para Anna Pujadas, profesora de la escuela Eina. El artículo "El dibujo analítico como método de interpretación del diseño gráfico" había sido consultado por 4.442 personas (a día de hoy ya son muchas más). Pujadas agradeció el premio que "el diseño gráfico empieza donde terminan las palabras", lo que nos obliga a profundizar con más insistencia sobre la naturaleza de esta disciplina. Por otro lado, se demostró la utilidad de la sección de Posters del Symposium para introducir a los jóvenes investigadores y a nuestros estudiantes en la reflexión crítica sobre el diseño, la publicidad y la comunicación gráfica.

En definitiva, por todo lo dicho, resulta claro que tenemos un reto muy duro para mejorar el nivel del encuentro del próximo año, pero en un encuentro que es de todos, no tengo la menor duda de que tanto la revista como el Symposium van a vivir un nuevo salto cualitativo que nos dejará nuevos recuerdos que valdrá la pena rememorar.

Para este número de grafica pesentamos cuatro artículos científicos, un ensayo y una reseña.

Los artículos científicos son de Sofia Escudero que nos presenta "Análisis de la estrategia visual de producto y marca en suplementos alimenticios para la tercera edad en marcas globales"; de Fernando Suárez-Carballo que nos ofrece un texto sobre "La enseñanza del diseño gráfico en los grados españoles vinculados a la comunicación publicitaria: perfil del profesorado, métodos docentes y competencias tecnológicas"; y Sheila Gonzalez-Mardones que nos muestra "Tipologías proyectuales en diseño gráfico. Hacia una clasificación funcional".

A éstos, les acompaña el ensayo de Lourdes Pilay Marco Neves sobre el "Diseño para la valoración del patrimonio cultural-gráfico de la ciudad". Finalizando los artículos de este número de grafica con la reseña de Francesc Morera Vidal sobre "Dejad que las máquinas vengan a mí"; una reflexión ontológica sobre qué hace humano al ser humano.

Así pues, solo nos queda agradecer a todos cuantos hacen posible este proyecto y esperar que este número sea del interés de los lectores. 
